\title{
Effect of Side-Chain Variation on Single-Crystalline Structures for Revealing the Structure-Property Relationships of Organic Solar Cells
}

\author{
Chen Yang ${ }^{a, b}$ (D) \\ Liu Yuan a \\ Ruimin Zhou ${ }^{\mathrm{a}, \mathrm{c}}$ \\ Zhen Wanga,b \\ Jianqi Zhang a (iD) \\ Yajie Zhang $^{\text {a }}$ \\ Kun Lu*a (D) \\ Zhixiang Wei $^{* a}$ (i) \\ ${ }^{a}$ CAS Key Laboratory of Nanosystem and Hierarchical Fabrication, CAS Center for \\ Excellence in Nanoscience, National Center for Nanoscience and Technology, \\ Beijing, China \\ weizx@nanoctr.cn; Ivk@nanoctr.cn \\ b School of Chemical Science, University of Chinese Academy of Sciences, Beijing, China \\ 'Sino-Danish Center for Education and Research, Sino-Danish College, University of \\ Chinese Academy of Sciences, Beijing, China
}

Received: 23.09.2019

Accepted after revision: 08.11.2019

DOI: 10.1055/s-0039-3402514; Art ID: om-19-0011-oa

License terms: CCO

(c) 2020. The Author(s). This is an open access article published by Thieme under the terms of the Creative Commons Attribution-NonDerivative-NonCommercial-License, permitting copying and reproduction so long as the original work is given appropriate credit. Contents may not be used for commercial purposes, or adapted, remixed, transformed or built upon. (https://creativecommons.org/licenses/by-nc-nd/4.0/).

Abstract The molecular stacking assembly in the active layer plays a significant role in the photovoltaic performance of organic solar cells (OSCs). Here, we report two new small molecular donors with different side chains, FBT-O and FBT-H, and their corresponding fullerene-based OSCs. A slight change in the side chains led to a big difference in the power conversion efficiencies (PCEs). Although the molecular structures of the two donors are similar to each other, PCEs of the devices based on FBT-O were almost three times higher than those of the devices based on FBT-H, with manifold short-circuit current density, fill factor, as well as three orders of magnitude enhancement in the hole mobility. The difference in their single crystal structures was thoroughly investigated, whereby the FBT-O exhibited better planarity leading to appropriate phase separation and domain size. Furthermore, two-dimensional grazing-incidence wide-angle $X$-ray scattering results of the blend films revealed that the two donors retained a similar stacking structure as compared to the single-crystal structures, thus, establishing a clear relationship between the molecular stacking structure and the device performance.

Key words side-chain modification, molecular stacking structure, hole mobility, single crystal

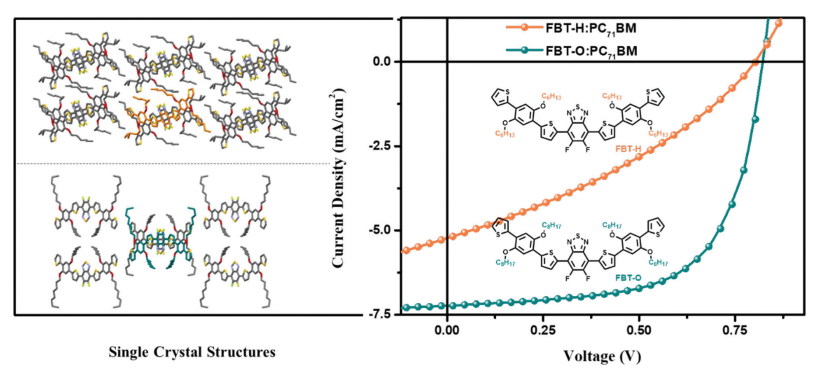

\section{Introduction}

Organic solar cells (OSCs) have attracted great attention because of several potential advantages, such as low cost, mechanical flexibility, lightweight, and tunable energy levels. ${ }^{1-5}$ Great progress has been made with the remarkable power conversion efficiencies (PCEs) of over 15\% based on polymer donors and nonfullerene acceptors in recent years. ${ }^{6-8}$ In the past decade, in order to obtain high-efficiency OSCs, polymer donor ${ }^{9-13}$ and small molecular donor materials ${ }^{1,14-18}$ have been extensively studied. In contrast to polymeric materials, small molecular donors have displayed great advantages in OSCs, such as well-defined molecular structures, high purity, and high reproducibility without batch-to-batch variation, ${ }^{19,20}$ which are beneficial to obtain single crystals. In addition, the crucial reason for rapid development in small molecular donors is that their molecular structures can be easily modified by $\pi$-conjugated backbone modifications, ${ }^{21-23}$ end-group modulation, ${ }^{24-27}$ and side-chain engineering. ${ }^{28-31}$ In bulk-heterojunction $(\mathrm{BHJ})$ OSCs, the molecular packing pattern and phase separation morphology in the photoactive layer have a big influence on exciton dissociation and charge transport. ${ }^{18,32}$ In the meantime, some research studies indicated that the length and the configuration of the end alkyl chain can realize the obvious transition of orientations from edge-on to face-on relative to the substrate. ${ }^{28}$ However, comparison studies of the length of the side chain with relevance to the molecular stacking are quite rare. In recent years, X-ray crystallographic structures of nonfullerene acceptors have been employed to investigate the relationship between molecular packing structure and photovoltaic performance of OSCs. ${ }^{33-37}$ Nevertheless, there is a lack of studies on single-crystal structures of 
small molecular donors and their correlation with photovoltaic performance, ${ }^{38,39}$ designing model compounds to study their crystal packing and film morphology, and then devising an effective and facile method for controlling the molecular orientation, all of which are of great importance.

Herein, we designed and synthesized two 5,6-difluorobenzo[c][1,2,5]thiadiazole $(\mathrm{dFBT})^{21}$-based small molecular donors with different side chains, named FBT-H and FBT-O (see Figure 1a). This design provides model compounds and allows us to study the crucial influence of the side chains on the crystal packing and intermolecular aggregation behavior in the active film. These two small molecular donors show identical UV-vis absorption spectra in chloroform solution, but FBT-O shows a comparatively larger red-shift in films than FBT-H. BHJ OSCs were then fabricated by employing FBT-H and FBT-O as donors and $\mathrm{PC}_{61} \mathrm{BM}$ and $\mathrm{PC}_{71} \mathrm{BM}$ as acceptors. PCEs of the devices based on FBT-O were almost three times higher than those of the FBT-H devices, with manifold short-circuit current density and fill factor (FF). The single-crystal structures, the phase-separation morphology, and molecular arrangements in the active layers were then compared, and different aggregation behaviors were observed. Furthermore, three orders of magnitude higher hole mobility for the FBT-O blend was observed as compared to the FBT-H blend films.

\section{Result and Discussion}

The synthesis routes of the two donor molecules are shown in Scheme S1 and the synthesis details are provided in the Supporting Information. The UV-vis absorption spectra for dilute solutions and thin films are shown in Figure 1. The molecules in dilute chloroform exhibited the same absorption in the visible region of 300-600 nm. However, distinct differences were observed for thin-film absorption, where FBT-O showed a larger red-shift with an absorption edge at $700 \mathrm{~nm}$, whereas FBT-H exhibited an absorption edge at $675 \mathrm{~nm}$, corresponding to optical bandgaps of 1.77 and
$1.84 \mathrm{eV}$, respectively. The energy levels of FBT-H and FBT-O were measured by cyclic voltammetry. The HOMO energy levels of FBT-H and FBT-O were calculated to be -5.25 and $-5.20 \mathrm{eV}$, respectively. The corresponding LUMO energy levels were -3.45 and $-3.50 \mathrm{eV}$, respectively.

Devices with a conventional structure of ITO/PEDOT:PSS/ active layer/Ca/Al were fabricated to investigate the photovoltaic properties. The current-voltage $(J-V)$ curves of optimized devices based on a donor:acceptor weight ratio of $1: 1$ with a total concentration of $20 \mathrm{mg} \mathrm{mL}^{-1}$ in chloroform solution with $0.2 \%$ 1,8-diiodooctane (DIO) as an additive are shown in Figure 2a, and the corresponding photovoltaic parameters are summarized in Table 1 . The FBT-H blend systems with $\mathrm{PC}_{61} \mathrm{BM}$ and $\mathrm{PC}_{71} \mathrm{BM}$ showed very low $\mathrm{FFs}$ of 33.50 and $33.60 \%$, with PCEs of 0.94 and $1.41 \%$, respectively. However, the FBT-Obased solar cells with $\mathrm{PC}_{61} \mathrm{BM}$ and $\mathrm{PC}_{71} \mathrm{BM}$ demonstrated improved PCEs of up to 3.01 and $3.82 \%$, with enhanced $J_{\mathrm{sc}}$ values of 5.75 and $7.24 \mathrm{~mA} \mathrm{~cm}^{-2}$, and a similar FF of around $64 \%$, respectively. The external quantum efficiencies (EQEs) of the devices for different blend systems are shown in Figure $2 b$, where the FBT-O-based devices exhibited higher EQE values. The $J_{\mathrm{sc}}$ values integrated from the EQEs are in good agreement with those obtained from the $J-V$ measurements. The obvious improvement in FF values of the FBT-O-based devices indicated less recombination in the charge generation and transport process, which gives rise to higher EQEs.

The space-charge-limited current (SCLC) method was employed to analyze the hole and electron mobilities of the blend systems. The electron-only structure devices based on the blend films of FBT-H:PC ${ }_{61} \mathrm{BM}, \mathrm{FBT}-\mathrm{H}: \mathrm{PC}_{71} \mathrm{BM}$, FBT-O: $\mathrm{PC}_{61} \mathrm{BM}$, and FBT-O:PC ${ }_{71} \mathrm{BM}$ showed mobilities of $2.88 \times 10^{-5}$, $4.07 \times 10^{-5}, \quad 6.00 \times 10^{-5}$, and $2.98 \times 10^{-5} \mathrm{~cm}^{2} \mathrm{~V}^{-1} \mathrm{~s}^{-1}$, respectively, indicating similar order of magnitudes (Figure S5, Supporting Information). However, the hole mobility values were calculated to be $5.13 \times 10^{-8} \mathrm{~cm}^{2} \mathrm{~V}^{-1} \mathrm{~s}^{-1}$, $5.66 \times 10^{-8}, 4.95 \times 10^{-5}$, and $4.19 \times 10^{-5} \mathrm{~cm}^{2} \mathrm{~V}^{-1} \mathrm{~s}^{-1}$, respectively, for the same set of devices (Figure S6, Supporting Information). Hence, a slight change in the length of the alkyl chain led to an obvious improvement in mobility. The mobility a
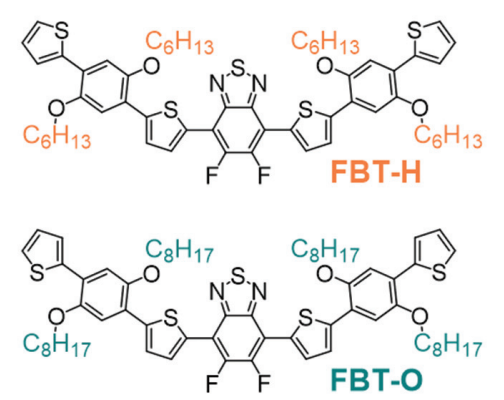

b

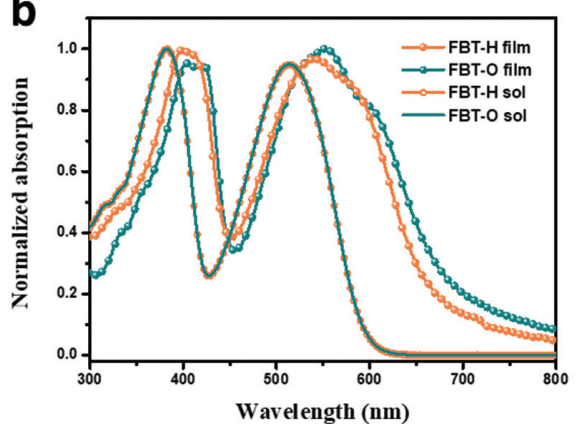

C

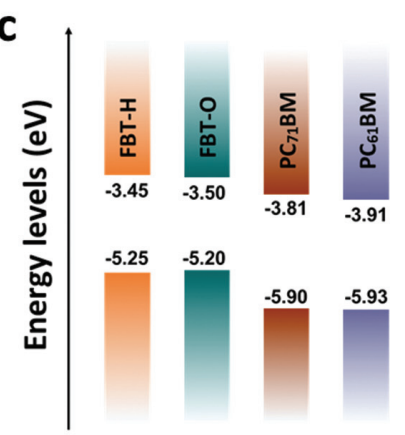

Figure 1 (a) Chemical structures of FBT-H and FBT-O, (b) UV-vis absorption spectra of FBT-H and FBT-O in chloroform solution and thin films, and (c) energy levels of $\mathrm{FBT}-\mathrm{H}$, FBT-O, $\mathrm{PC}_{71} \mathrm{BM}$, and $\mathrm{PC}_{61} \mathrm{BM}$. 

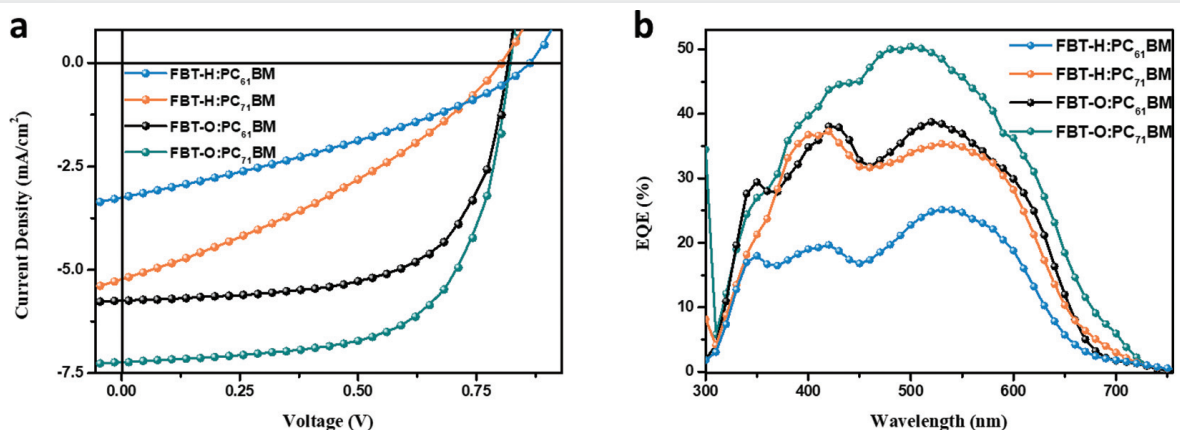

Figure 2 (a) The J-V curves under AM 1.5 G irradiation, and (b) the corresponding EQEs for solar cells.

Table 1 Photovoltaic performance of devices based on the two donors

\begin{tabular}{|c|c|c|c|c|c|c|c|}
\hline Donor:acceptor & Additive & $V_{\text {oc }}[\mathrm{V}]$ & $J_{\mathrm{sc}}\left[\mathrm{mA} \mathrm{cm}{ }^{-2}\right]$ & $\mathrm{FF}[\%]$ & PCE [\%] & $\mu_{\mathrm{h}}\left[\mathrm{cm}^{2} \mathrm{~V}^{-1} \mathrm{~s}^{-1}\right]$ & $\mu_{\mathrm{e}}\left[\mathrm{cm}^{2} \mathrm{~V}^{-1} \mathrm{~s}^{-1}\right]$ \\
\hline FBT-H:PC ${ }_{61} B M$ & $0.2 \% \mathrm{DIO}$ & 0.86 & 3.27 & 33.50 & 0.94 & $5.13 \times 10^{-8}$ & $2.88 \times 10^{-5}$ \\
\hline $\mathrm{FBT}-\mathrm{H}: \mathrm{PC}_{71} \mathrm{BM}$ & $0.2 \%$ DIO & 0.80 & 5.24 & 33.60 & 1.41 & $5.66 \times 10^{-8}$ & $4.07 \times 10^{-5}$ \\
\hline FBT-O:PC ${ }_{61}$ BM & $0.2 \% \mathrm{DIO}$ & 0.82 & 5.75 & 63.78 & 3.01 & $4.95 \times 10^{-5}$ & $6.00 \times 10^{-5}$ \\
\hline FBT-O:PC ${ }_{71}$ BM & $0.2 \% \mathrm{DIO}$ & 0.83 & 7.24 & 63.98 & 3.82 & $4.19 \times 10^{-5}$ & $2.98 \times 10^{-5}$ \\
\hline
\end{tabular}

values are summarized in Table 1, in which the results indicate a more balanced transport and higher mobility values from the FBT-O blend systems, which can explain the striking differences of $J_{\mathrm{sc}}$ and FF.

To further understand the relationship between the morphologies of active layers and device photovoltaic performance, atomic force microscopy (AFM), transmission electron microscopy (TEM), and grazing-incidence wideangle X-ray scattering (GIWAXS) were employed to investigate the morphology of the blend films. The AFM height images are shown in Figure 3, in which the FBT-H: $\mathrm{PC}_{61} \mathrm{BM}$ and $\mathrm{FBT}-\mathrm{H}: \mathrm{PC}_{71} \mathrm{BM}$ blend films exhibit rougher and more disorderly surfaces with root-mean-square (RMS) roughness values of 2.89 and $8.45 \mathrm{~nm}$, respectively.
However, for the FBT-O:PC ${ }_{61} \mathrm{BM}$ and FBT-O:PC ${ }_{71} \mathrm{BM}$ blend films, apparent microbelts and appropriate phase separation can be seen, as smooth surfaces with RMS values of 2.78 and $2.64 \mathrm{~nm}$, respectively, were observed. Furthermore, as can be seen from the TEM images, the FBT-H:PC ${ }_{61} \mathrm{BM}$ and FBT-H:PC ${ }_{71} \mathrm{BM}$ blend films show similar arborization morphologies and large phase separation. However, compared to the FBT-H blend systems, the FBT-O:PC ${ }_{61} \mathrm{BM}$ and FBT-O:PC ${ }_{71} \mathrm{BM}$ blend films show completely different morphologies. Interconnected phase-separated domains with microbelt structures of 15-20 nm width and around $200 \mathrm{~nm}$ length were formed in the FBT-O blend systems, which are consistent with the AFM results. These microbelt structures with a high degree of molecular ordering were
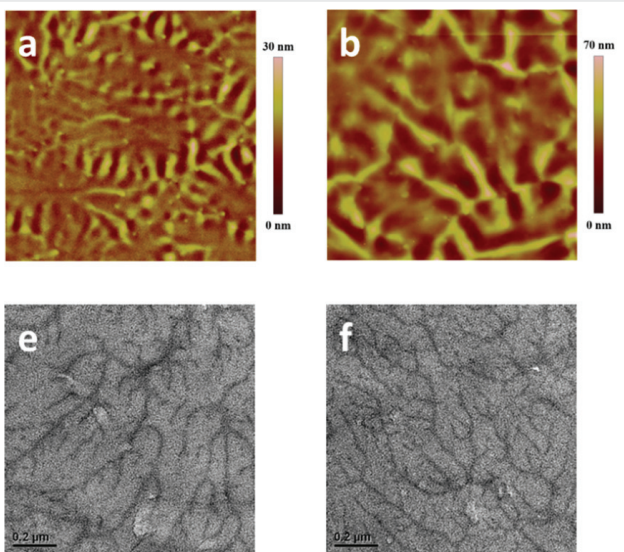
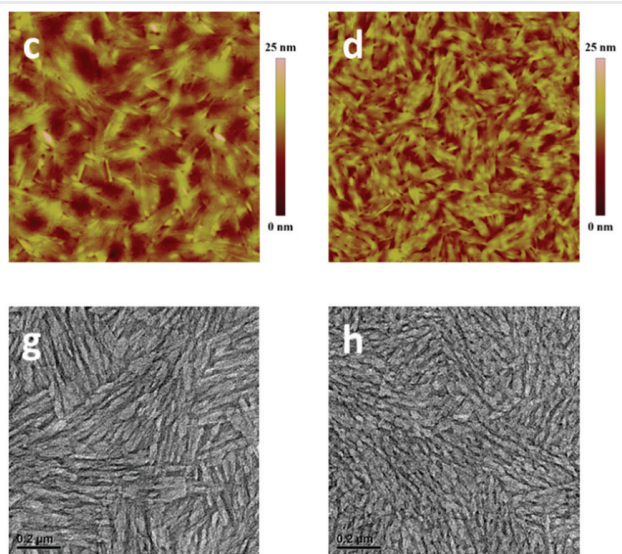

Figure 3 The AFM height and TEM images of optimized FBT-H:PC 61 BM (a, e), FBT-H:PC ${ }_{71} B M(b, f), F B T-O: P C_{61} B M(c, g)$, and FBT-O:PC ${ }_{71} B M(d, h)$. 
beneficial for exciton diffusion and charge transport, one of the reasons for their higher FF and $J_{\text {sc }}$.

Solid-state structures play a critical role in understanding the molecular packing and are of great importance for the rational design of new donor molecules. Nevertheless, there is a striking paucity of single-crystal diffraction data. To gain detailed insight into FBT-H and FBT-O molecular packing, consequently, suitable single crystals of FBT-H and FBT-O were grown via slow diffusion and the corresponding diffraction data were acquired (see the Supporting Information for complete details).

The crystal structures of FBT-H and FBT-O are shown in Figure 4. Notably, the single-crystal structures of the two donors revealed a co-planar structure of the conjugated backbone, which interact with each other via $\pi$-stacking with a distance of $3.49 \AA$, which dominates the crystal packing. The thiophene rings of both molecules which connect with the dFBT exhibit a cis-conformation and the two molecules exhibit intermolecular $\mathrm{S}-\mathrm{N}$ interaction with a distance of $2.75 \AA$, which is closer than the sum of the $S$ and $\mathrm{N}$ van der Waals radii (3.25 $\AA$ ). In crystals of FBT-H, molecules crystallize in triclinic unit cells, leading to the torsion angle between the end-group thiophene and the dFBT core to be close to $21.28^{\circ}$. However, for FBT-O, molecules crystallize in the monoclinic unit cells, and the torsion angle between the end-group thiophene and the dFBT core is close to $8.82^{\circ}$, indicating better planarity in one direction of FBT-O, which should be beneficial for charge transportation between different connected molecules.

GIWAXS measurements and analyses were conducted to investigate how the alkyl chain influences the stacking structures of the active layer. Figure 5 shows the twodimensional (2D) GIWAXS scattering patterns of the blend films as cast on bare Si substrates. In Figure 5a it can be seen that the 2D GIWAXS result corresponding to the FBT-H film displays distinct Bragg rods, has distinct lamellar packing at
$0.32 \AA^{-1}$, and the corresponding interlamination distance is 19.63 Å. What's more, FBT-H exhibits a tighter alkyl packing at $0.50 \AA^{-1}$ and a $\pi-\pi$ stacking peak at $1.75 \AA^{-1}$ with distances of 12.57 and $3.59 \AA$, respectively. The strong diffraction peak located at $0.58 \AA^{-1}$ can be assigned to the (011) lamellar stacking. These values are fairly similar to that of the corresponding bulk single-crystal spacing. From Figure 5d, we can see that the 2D GIWAXS result corresponding to the FBT-O film displays distinct diffraction spots, indicating a high degree of molecular ordering. Notably, FBT-O gives a sharp lamellar packing at $0.35 \AA^{-1}$ and a tighter alkyl packing at $0.43 \AA^{-1}$, and the corresponding distances are 17.95 and $14.61 \AA$, corresponding to the values of the single crystal: $d$ $(002)=17.95 \AA$ and $d(010)=15.45 \AA$. Likewise, the $\pi-\pi$ stacking peak of FBT-O was located at $1.76 \AA^{-1}$ with a distance of $3.57 \AA$ A. For the FBT-H:PC ${ }_{61} B M$ and FBT-H:PC ${ }_{71} B M$ blend films, the scattering pattern exhibited similar diffraction peaks at $0.32,0.47$, and $0.58 \AA^{-1}$, which are assigned to the (001), (010), and (011) lamellar stacking scattering of FBT-H, respectively. It is apparent that the pure FBT-H and its fullerene blend films arrange in nearly the same way on the substrate and share the same packing motif. However, the $\pi-\pi$ stacking peak is weakened compared to the pristine FBT-H film, indicating a lower degree of molecular ordering. For FBT$\mathrm{O}$ :fullerene blend films, the diffraction spots of the blend films are nearly identical to those of pure FBT-O and the diffraction peaks are located at 0.35 and $0.41 \AA^{-1}$. In contrast to the FBT-H blend films, the interlamellar spacing $(17.95 \AA)$ is shorter than

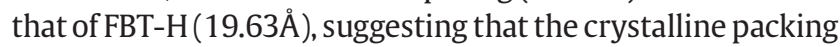
of FBT-O is more efficient for charge transport between layers.

\section{Conclusions}

In summary, two small molecular donors with different alkyl chains, namely FBT-H and FBT-O, were synthesized.
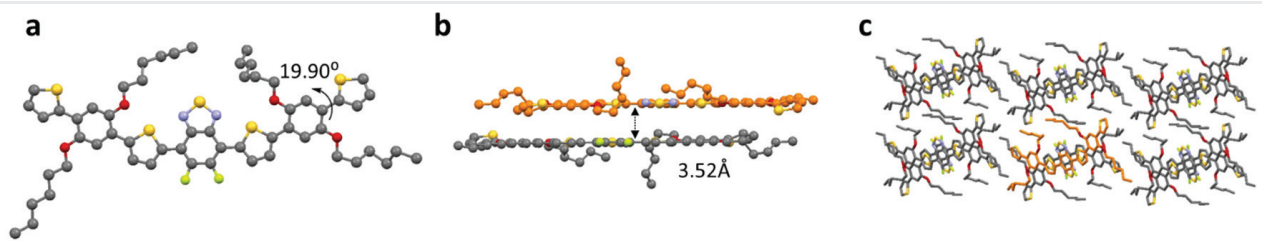

d

e

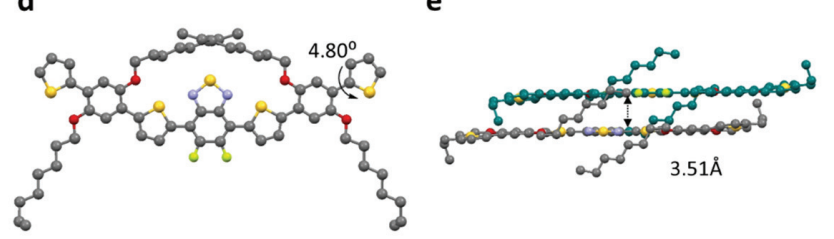

f

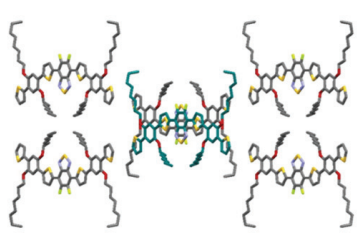

Figure 4 X-ray crystallographic structures of FBT-H and FBT-O, molecular conformation (a, d); $\pi$-stacking dimers of FBT-H and FBT-O in their singlecrystal view along the $\pi$-stacking direction (b, e) and the out-of-plane direction (c, f). 

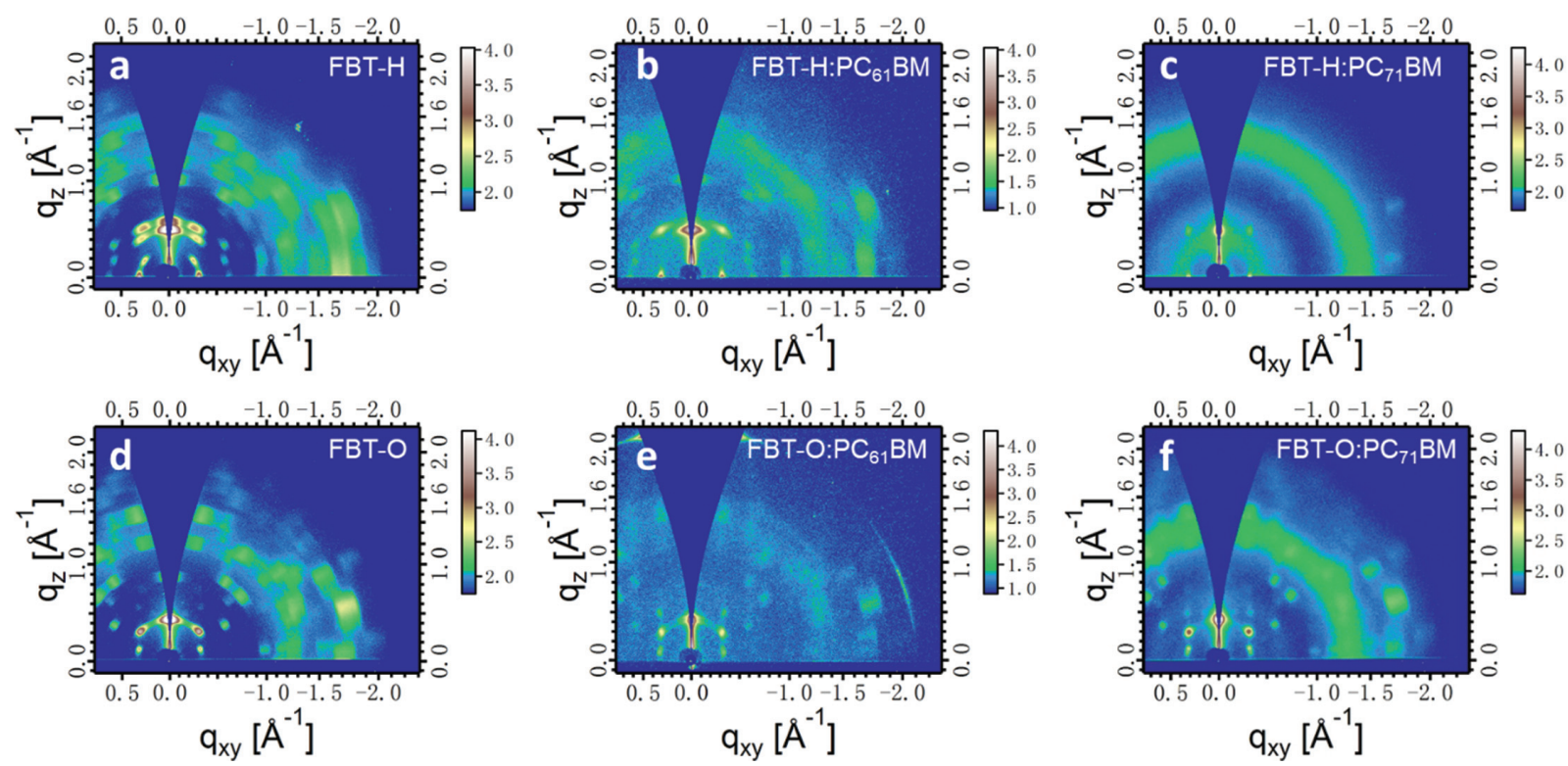

Figure 5 2D GIWAXS patterns of neat FBT-H (a), FBT-H:PC ${ }_{61} B M(b)$, FBT-H:PC 71 BM (c), neat FBT-O (d), FBT-O:PC $C_{61} B M(e)$, and FBT-O:PC $C_{71} B M(f)$.

Particularly, the photovoltaic performance of FBT-O:fullerene blend solar cells demonstrate an obvious enhancement in $J_{\mathrm{sc}}$ and FF in contrast to those of FBT-H:fullerene blend solar cells. Furthermore, the single crystals of FBT-H and FBT-O have been successfully obtained and thoroughly investigated. The two single crystals have completely different packing structures and the results exhibited that FBT-O has better planarity and shorter interlamellar spacing, which are conducive to intermolecular charge transport, as demonstrated by the distinct difference in hole mobility. Furthermore, the GIWAXS results of the two blend systems show similar molecular packing motifs compared to bulk single crystals. Specifically, this work indicates that modifying the alkyl chains of smallmolecule donors is an effective and powerful strategy for adjusting the molecular packing structure.

\section{Experimental Section}

\section{Materials}

The detailed synthetic methods of FBT-H and FBT-O are shown in the Supporting Information.

\section{General Characterization}

${ }^{1} \mathrm{H}$ NMR spectra were measured on a Bruker DMX-400 NMR spectrometer using $d$-chloroform as the solvent and trimethylsilane as the internal standard. Mass spectra (MALDI-TOF MS) were measured on a Micromass GCT-MS spectrometer. Ultraviolet-visible absorption was measured on a JASCO V-570 spectrophotometer. The cyclic voltammetry measurement was carried out at an electrochemical workstation (VMP3 Biologic, France) in an acetonitrile solution of $0.1 \mathrm{M}$ tetrabutylammonium phosphorus hexafluoride $\left(\mathrm{Bu}_{4} \mathrm{NPF}_{6}\right)$ with a Pt electrode coated with FBT-H and FBT-O films as a working electrode, and a Pt plate and an $\mathrm{Ag} / \mathrm{Ag}^{+}$electrode were used as the counter electrode and reference electrode, respectively. The SCLC method was used to measure hole and electron mobilities, and the current density-voltage $(J-V)$ curves were tested using a Keithley 2400 source-measure unit in the dark. A Tecnai G2 F20 UTWIN TEM instrument was employed for TEM tests. The AFM images were measured on a Bruker FastScan atomic force microscope. And GIWAXS measurements were conducted at the XEUSS SAXS/WAXS beamline.

\section{Device Fabrication and Characterization}

The devices were fabricated with a conventional structure of ITO/PEDOT:PSS/active layer/Ca/Al. The ITOcoated glass was cleaned with detergent, DI water, acetone, and isopropyl alcohol for $30 \mathrm{~min}$ at each step by ultrasonic treatment. A thin layer of PEDOT:PSS was spin-coated on a precleaned ITO-coated glass at 3,500 rpm and dried subsequently at $150{ }^{\circ} \mathrm{C}$ for $15 \mathrm{~min}$. Then the active blend layer was spin-coated from its chloroform solution onto the PEDOT:PSS layer at 2,500 rpm in a nitrogen-filled glove box. Finally, the top $\mathrm{Ca}$ and $\mathrm{Al}$ layers were deposited at a pressure of about $1 \times 10^{-5}$ Pa with a thickness of 20 and $100 \mathrm{~nm}$, 
respectively. The area of the device was $4 \mathrm{~mm}^{2}$, and the $J-V$ curves were measured under AM $1.5\left(100 \mathrm{Mw} \mathrm{cm}^{-2}\right)$ using a Newport Thermal Oriel 91159 A solar simulator. EQE curves were tested using an Oriel Newport system (Model 66902) in air with a standard Si diode.

\section{Crystallographic Data}

Single-crystal structure of FBT-H: 1965714. Singlecrystal structure of FBT-O: 1965715.

\section{Funding Information}

The authors acknowledge the financial support from the National Natural Science Foundation of China (Grant Nos. 21822503, 21534003, 21125420, and 91427302), the Ministry of Science and Technology of China (Grant Nos. 2016YFA0200704 and 2016YFF0203803), the Beijing Nova Program (Grant No. Z17110001117062), and the Chinese Academy of Sciences.

\section{Acknowledgment}

The authors thank Dr. Ming Li of Tsinghua University for the refinement of single-crystal structure data.

\section{Supporting Information}

Supporting information for this article is available online at https://doi.org/10.1055/s-0039-3402514.

\section{References}

(1) Deng, D.; Zhang, Y-J.; Zhang, J-Q.; Wang, Z-Y.; Zhu, L-Y.; Fang, J.; Xia, B-Z.; Wang, Z.; Lu, K.; Ma, W.; Wei,Z-X.Nat.Commun. 2016, 7, 13740.

(2) Yuan, L.; Lu, K.; Xia, B-Z.; Zhang, J. Q.; Wang, Z.; Wang, Z-Y.; Deng, D.; Fang, J.; Zhu, L-Y.; Wei, Z-X. Adv. Mater. 2016, 28, 5980.

(3) Yuan, L.; Zhao, Y-F.; Zhang, J-Q.; Zhang, Y-J.; Zhu, L-Y.; Lu, K.; Yan, W.; Wei, Z-X. Adv. Mater. 2015, 27, 4229.

(4) Deng, D.; Zhang, Y.; Wang, Z.; Wu, Q.; Ma, W.; Lu, K.; Wei, Z-X. Asian J. Org. Chem. 2018, 7, 558.

(5) Li, H.; Wu, Q.; Zhou, R-M.; Shi, Y-N.; Yang, C.; Zhang, Y. J.; Zhang, J-Q.; Zou, W-J.; Deng, D.; Lu, K.; Wei, Z-X. Adv. Energy Mater. 2019, 9, 1803175.

(6) Che, X-Z.; Li, Y-X.; Qu, Y.; Forrest, S. R. Nat. Energy 2018, 3, 422.

(7) Yuan, J.; Zhang, Y-Q.; Zhou, L-Y.; Zhang, G-C.; Yip, H-L.; Lau, T-K.; Lu, X-H.; Zhu, C.; Peng, H-J.; Johnson, P. A.; Leclerc, M.; Cao, Y.; Ulanski, J.; Li, Y. F.; Zou, Y-P. Joule 2019, 3, 1140.

(8) Meng, L-X.; Zhang, Y-M.; Wan, X-J.; Li, C-X.; Zhang, X.; Wang, YB.; Ke, X.; Xiao, Z.; Ding, L-M.; Xia, R-X.; Yip, H-L.; Cao, Y.; Chen, Y-S. Science 2018, 361, 1094.
(9) Zhang, M-J.; Guo, X.; Ma, W.; Ade, H.; Hou, J-H. Adv. Mater. 2015, 27, 4655.

(10) Li, S-S.; Ye, L.; Zhao, W-C.; Yan, H-P.; Yang, B.; Liu, D-L.; Li, W-N.; Ade, H.; Hou, J-H. J. Am. Chem. Soc. 2018, 140, 7159.

(11) Xu, X-P.; Yu, T.; Bi, Z-Z.; Ma, W.; Li, Y.; Peng, Q. Adv. Mater. 2018, 30, 1703973.

(12) Liu, D-L.; Yang, B.; Jang, B-M.; Xu, B-W.; Zhang, S-Q.; He, C.; Woo, H. Y.; Hou, J-H. Energy Environ. Sci. 2017, 10, 546.

(13) Wu, Y-N.; An, C-B.; Shi, L-L.; Yang, L-Y.; Qin, Y-P.; Liang, N-N.; He, C.; Wang, Z-H.; Hou, J-H. Angew. Chem. Int. Ed. 2018, 57, 12911.

(14) Li, H.; Zhao, Y-F.; Fang, J.; Zhu, X-W.; Xia, B-Z.; Lu, K.; Wang, Z.; Zhang, J-Q.; Guo, X-F.; Wei, Z-X. Adv. Energy Mater. 2018, 8, 1702377.

(15) Deng, D.; Yang, Y.; Zou, W-J.; Zhang, Y-J.; Wang, Z.; Wang, Z-Y.; Zhang, J-Q.; Lu, K.; Ma, W.; Wei, Z-X. J. Mater. Chem. A 2018, 6, 22077.

(16) Zhou, R-M.; Xia, B-Z.; Li, H.; Wang, Z.; Yang, Y.; Zhang, J-Q.; Laursen, B. W.; Lu, K.; Wei, Z-X. Front. Chem. 2018, 6, 384.

(17) Yang, L-Y.; Zhang, S-Q.; He, C.; Zhang, J-Q.; Yang, Y.; Zhu, J.; Cui, Y.; Zhao, W-C.; Zhang, H.; Zhang, Y.; Wei, Z-X.; Hou, J. H. Chem. Mater. 2018, 30, 2129.

(18) Li, X-X.; Wang, Y.; Zhu, Q-L.; Guo, X.; Ma, W.; Ou, X-M.; Zhang, MJ.; Li, Y-F. J. Mater. Chem. A 2019, 7, 3682.

(19) Qiu, B-B.; Chen, S-S.; Xue, L-W.; Sun, C-K.; Li, X-J.; Zhang, Z-G.; Yang, C-D.; Li, Y-F. Front. Chem. 2018, 6, 413.

(20) Guo, J.; Bin, H-J.; Wang, W.; Chen, B-C.; Guo, J.; Sun, R.; Zhang, ZG.; Jiao, X-C.; Li, Y-F.; Min, J. J. Mater. Chem. A 2018, 6, 15675.

(21) Yuan, L.; Lu, K.; Xia, B-Z.; Zhang, J-Q.; Wang, Z.; Wang, Z-Y.; Deng, D.; Fang, J.; Zhu, L-Y.; Wei, Z-X. Adv. Mater. 2016, 28, 5980.

(22) Yang, L-Y.; Zhang, S-Q.; He, C.; Zhang, J-Q.; Yao, H-F.; Yang, Y.; Zhang, Y.; Zhao, W-C.; Hou, J-H. J. Am. Chem. Soc. 2017, 139, 1958.

(23) Wei, H.; Chen, W-C.; Han, L-L.; Wang, T.; Bao, X-C.; Li, X-Y.; Liu, J.; Zhou, Y-H.; Yang, R-Q. Chem. Asian J. 2015, 10, 1791.

(24) Duan, R-M.; Cui, Y.; Zhao, Y-F.; Li, C.; Chen, L.; Hou,J-H.; Wagner, M.; Baumgarten, M.; He, C.; Muellen, K. ChemSusChem 2016, 9, 973.

(25) Duan, T-N.; Tang, H.; Liang, R-Z.; Lv, J.; Kan, Z-P.; Singh, R.; Kumar, M.; Xiao, Z-Y.; Lu, S-R.; Laquai, F. J. Mater. Chem. A 2019, 7, 2541.

(26) Kim, S. W.; Lee, Y. J.; Lee, Y. W.; Koh, C. W.; Lee, Y.; Kim, M. J.; Liao, K.; Cho, J. H.; Kim, B. J.; Woo, H. Y. ACS Appl. Mater. Interfaces 2018, 10, 39952.

(27) Chen, X-J.; Feng, H-R.; Lin, Z-J.; Jiang, Z-W.; He, T.; Yin, S-C.; Wan, X-J.; Chen, Y-S.; Zhang, Q.; Qiu, H-Y. Dyes Pigm. 2017, 147, 183.

(28) Yang, L-Y.; Zhang, S-Q.; He, C.; Zhang, J-Q.; Yang, Y.; Zhu, J.; Cui, Y.; Zhao, W-C.; Zhang, H.; Zhang, Y.; Wei, Z-X.; Hou, J-H. Chem. Mater. 2018, 30, 2129.

(29) Hong, J.; Sung, M-J.; Cha, H.; Park, C. E.; Durrant, J. R.; An, T. K.; Kim, Y. H.; Kwon, S. K. ACS Appl. Mater. Interfaces 2018, 10, 36037.

(30) Yao, X.; Shao, W.; Xiang, X.; Xiao, W-J.; Liang, L.; Zhao, F-G.; Ling, J.; Lu, Z-Q.; Li, J-J.; Li, W-S. Org. Electron. 2018, 61, 56.

(31) Kumari, T.; Lee, S. M.; Lee, K. C.; Cho, Y.; Yang, C. Adv. Energy Mater. 2018, 8, 1800616.

(32) Min, J.; Kwon, O. K.; Cui, C-H.; Park, J. H.; Wu, Y.; Park, S. Y.; Li, Y-F.; Brabec, C. J. J. Mater. Chem. A 2016, 4, 14234.

(33) Aldrich, T. J.; Matta, M.; Zhu, W.; Swick, S. M.; Stern, C. L.; Schatz, G. C.; Facchetti, A.; Melkonyan, F. S.; Marks, T. J. J. Am. Chem. Soc. 2019, 141, 3274.

(34) Qu, J-F.; Chen, H.; Zhou, J-D.; Lai, H-J.; Liu, T.; Chao, P-J.; Li, D-N.; Xie, Z-Q.; He, F.; Ma, Y-G. ACS Appl. Mater. Interfaces 2018, 10, 39992. 
(35) Swick, S. M.; Zhu, W.; Matta, M.; Aldrich, T. J.; Harbuzaru, A.; Lopez Navarrete, J. T.; Ponce Ortiz, R.; Kohlstedt, K. L.; Schatz, G. C.; Facchetti, A.; Melkonyan, F. S.; Marks, T. J. Proc. Natl. Acad. Sci. U.S.A. 2018, 115, E8341.

(36) Shi, X-L.; Zuo, L-J.; Jo, S. B.; Gao, K.; Lin, F.; Liu, F.; Jen, A. KY. Chem. Mater. 2017, 29, 8369.

(37) Han, G-C.; Guo, Y.; Song, X-X.; Wang, Y.; Yi, Y-P.J. Mater. Chem. C 2017, 5, 4852 .
(38) McDowell, C.; Narayanaswamy, K.; Yadagiri, B.; Gayathri, T.; Seifrid, M.; Datt, R.; Ryno, S. M.; Heifner, M. C.; Gupta, V.; Risko, C.; Singh, S. P.; Bazan, G. C. J. Mater. Chem. A 2018, $6,383$.

(39) Sun, K.; Xiao, Z-Y.; Lu, S-R.; Zajaczkowski, W.; Pisula, W.; Hanssen, E.; White, J. M.; Williamson, R. M.; Subbiah, J.; Ouyang, J.; Holmes, A. B.; Wong, W. WH.; Jones, D. J. Nat. Commun. 2015, 6,6013 